\title{
Sur certaines espèces de Lumbriculidae (Annelida : Oligochaeta) du nord de la péninsule ibérique
}

Pilar Rodriguez

Mots clés : Oligochaeta, Lumbriculidae, taxonomie, nouvelle sous-espèce, tératologie, Espagne.

Ce travai I donne une xérie de caractères morphométriques de cinq espéces de lumbriculides très communes dans les rivières du Nord de la Péninsule Ibérique. On y décrit une nouvelle variation du système reproducteur de Lumbricultus variegatus, des phénomènes tératologiques chez Stylodrilus heringianus et $S$. lemani, ainsi que la nouvelle sous-espèce Trichodrilus strandi campoyi.

On some species of Iumbriculidae (Annelida : Oligochaeta) from the North of the Iberian Peninsula.

Keywords : Oligochaeta, Lumbriculidae, taxonomy, new sub-species, teratology, Spain.

Morphometric characters of five lumbriculid species, common in the North lberian rivers, are given. A new variation on the reproductive system of Lumbriculus variegatus, teratological phenomena in Stylodrilus heringianus and S. lemant, and a new subspecies Trichodrilus strandi campoyi are described.

\section{Introduction}

Les lumbriculides constituent une composante essentielle de la communauté d'oligochètes aquatiques des eaux courantes, non polluées, de la Péninsule Ibérique. Cette région a étê peu étudiée et jusqu'à présent seulement sept espèces de lumbriculides y ont été trouvées. De ces espèces, deux étaient nouvelles pour la Science : Stylodrilus glandulosus Giani et Martinez-Ansemil, 1984 et Tricho. drilus diversisetosus Rodriguez, 1986. Cette dernière espèce est cavernicole. Ces résultats suggèrent l'importance de cette région géographique pour les études taxonomiques des oligochètes, et spécialement des lumbriculides.

Le présent travail constitue une étude morphométrique des populations de lumbriculides du Pays Basque et des régions avoisinantes (sauf

1. Laboratorio de Zoologia. Facultad de Ciencias. Universidad del País Vasco. Apdo. 644. Bilbao 48080 Espagne
$T$. diversiserosus) et apporte de nouveaux compléments à la diagnose des espèces.

\section{Matériel et méthodes}

Les vers ont été étudiés à partir d'exemplaires récoltés dans un grand nombre de localités : 103 stations de prélèvement situées dans 16 bassins de notre région d'étude (Bidasoa, Oiartzun, Urumea, Oria, Urola, Deba, Artibai Lea, Laga, Oca, Estepona, Butrón, Gobelas, Nervión, Asón et Ebro).

Les animaux ont été fixés au formaldéhyde à 10 $\%$ et conservés en alcool à $70 \%$. Ils ont été colorés à l'hématoxyline d'Erlich, avant d'être disséqués et montés au baume du Canada.

\section{Résultats}

Lumbriculus variegatus (Müller, 1774).

Fig. 1. 




Fig. 1. Lumbricultes variegatus (Müller) : A. Appareil reproducteur. B. Schémas de la distribution des élêments de l'appareil reproducteur sur différents individus. a : atrium, sp : spermatheque, ef : entonnoir fernelle, o: ovaire, $\mathbf{t}:$ testicule, or : ovaire réduit, $t r:$ testicule réduit, $\mathrm{ps}$ : pore de la spermathèque, $\mathrm{psf}:$ pore fermé de la spermathèque, spr : spermathèque réduite, $X$ : segment $X$. 
Matêriel étudié : 1 exemplaire complètement mûr. 5 exemplaires partiellement mûrs, colorés à l'hématoxyline d'Erlich, disséqués et montés au baume du Canada. Localités: Munguia, R. Butrớn, 30TWN 119018,9 Mars 1981. R. Artibai, 30 TWN 413904,25 nov. 1985 ,

\section{REMARQUES ANATOMIQUES}

Longueur : $\mathbf{3 0 - 4 0 ~} \mathrm{mm}$. Diamètre du corps environ $1 \mathrm{~mm}$. (matériel fixé au formaldéhyde). Nombre de segments : 80 à 120 . Les soies sont bifides tout au long du corps. Longueur des soies antérieures dorsales: 165-207. $3 \mu \mathrm{m}$ : les antérieures ventrales: 175.6-207.3 $\mu \mathrm{m}$; les postérieures : $168-195 \mu \mathrm{m}$.

Les glandes pharyngiennes sont sítuées sur les segments IV à VII, dorsalement et ventralement par rapport au tube digestif. Les cellules chloragogènes débutent en VIII. La position des gonades et celle de l'appareil reproducteur ont été schématisées sur la figure 1. Nous donnons ci-dessous les mesures prises sur le seul individu mature qui avait des spermatozoides dans les spermathèques. Atrium piriforme à tubulaire, $1236 \mu \mathrm{m}$ de long et $230 \mu \mathrm{m}$ de large, orienté vers l'arrière. La partie distale est droite et très musculeuse. L'épithélium atrial est extrêmement fin avec une couche musculaire d'environ $18.6 \mu \mathrm{m}$ d'épaisseur. L'ampoule atriale est complètement recouverte par des cellules prostatiques. Nous n'avons pas vu les spermiductes et les entonnoirs séminaux. La position des pores des spermathèques est typique, c'est-à-dire, dorso-latérale, sur la ligne des soies dorsales.

\section{Discuș̦ion}

Mrazek (1906) a étudié l'appareil génital de Lumbriculus variegatus. Il a fait une importante description des variations relatives au nombre et à la position des éléments de l'appareil mâle et femelle qui peuvent se présenter dans les populations. Cook (1971) a révisé le genre en proposant un caractère polytypique pour $L$. variegatus, avec 5 sous-especes géographiques. Chaque sous-espèce présente une certaine fréquence d'apparition de l'appareil mâle sur différents segments, ainsi qu'un nombre de paires de gonades caractéristique. En suivant la classification de Cook, notre matériel peut être at tribué a la sous-espece inconstans (Smith, 1895), car la plupart du matériel présente les pores mâles en $X, 2$ paires de testicules et deux paires d'ovaires. Quelquefois les gonades sont très peu développées. Cette sous-espèce n'avait été signalée jusqu'à maintenant qu'en Amérique du Nord.

Sokolskaya (1976) considère comme probablement correcte l'inclusion de L inconstans (Smith) dans $L$. variegatus (Müller). Au contraire. Holmquist (1976) croit convenable de réétablir le genre Thinodrilus Smith pour cette espece et celles qui présentent des entonnoirs séminaux et spermiductes en position pré- et/ou post-atriale. Lumbriculus inclurait uniquement les populations qui ont ces éléments en position post-atriale. Nos exemplaires, malheureusement, sont dépourvus d'entonnoirs et de spermiductes et nous ne pouvons donc pas apporter de nouvelles données à cette intéressante discussion. Le fait que ces organes puissent être absents avait été signalé auparavant par Mrazek (1906) et Timm (1979). L'existence de trois paires d'ovaires dans un individu avec l'appareil génital mâle développé est un fait nouveau à notre connaissance. Mrazek (1906) avait décrit sur les figures E13 et E16 de son vaste travail une série de spécimens avec trois paires d'ovaires; il les qualifie de "Reine weibschen" (femelles pures), car ils ne présentent aucune trace d'appareil mâle. Enfin, le manque de symétrie dans la disposition des éléments génitatux dans presque tout le matériel étudié est très remarquable.

Stylodrilus heringianus Claparède, 1862

Fig. 2 et 4B, 4C.

\section{REMARQUES ANATOMIQUES}

Longueur : environ $20 \mathrm{~mm}$. Diamètre (dans la région du clitellum) : $0.6 \mathrm{~mm}$. Nombre de segments : 52-83. Double annulation à partir du segment IV. Glandes pharyngiennes dans les segments V à VII, disposées dorsalement et ventralement par rapport au tube digestif. Les cellules chloragogènes débutent en VI. La plupart des soies sont bifides, bien que les premiers segments puissent présenter quelques soies à pointe simple, dues probablement à l'usure par frottement (Hrabe 1970). Les soies des faisceaux dorsaux antérieurs ont 86-124 $\mu \mathrm{m}$ de longueur, les antérieures ventrales $96-124 \mu \mathrm{m}$ et les postérieures $70-110 \mu \mathrm{m}$.

La position des gonades et de l'appareil reproducteur coincident avec la description de Cook (1971). Le clitellum occupe les segments Xà XII. La vésicule séminale atteint le segment IX vers l'avant et le segment XV vers l'arrière. L'ovisac peut 




Fig. 2. Stylodrilus heringianus Clap. Détail de l'atrium (a) et du pénis (p).

s'étendre jusqu'en XVI. L'atrium présente une ampoule globulaire de $130-200 \mu \mathrm{m}$ de longueur et de 70-90 $\mu \mathrm{m}$ de diamètre, avec une couche musculaire de 9.40-14 $\mu \mathrm{m}$ d'épaisseur. Les spermiductes mesurent environ $390-488 \mu \mathrm{m}$ de longueur et 22 à $32 \mu \mathrm{m}$ de diamètre. Le canal déférent postérieur pénètre en XI, où il forme une boucle. Distalement l'atrium présente un pénis de 79-325 $\mu \mathrm{m}$ de long. Ce pénis se trouve dans un long pli de la paroi du corps, dirigé vers l'arrière obliquement ou bien plaqué à la paroi du corps. Il présente une capacité très limitée de sortir de son pli (fig. 4). A la base du pénis existe un anneau musculaire. Les spermathèques sont constituées par un canal (220-377 $\mu \mathrm{m}$ de long) et une ampoule souvent hémisphèrique $(240-360 \mu \mathrm{m}$ de diamètre). La paroi de l'ampoule est fortement vacuolisée sur les individus complètement mûrs. La lumière de l'ampoule contient les spermatozoïdes avec du matériel indéterminé et des petits cristaux.

\section{DISCUSSION}

Quelques exemplaires de notre collection montrent des caractères qui pourraient les faire identifier comme $S$. brachystilus Hrabe, 1929 (fig. 4C). Ces exemplaires manquent de spermatozoides dans les spermathèques. La paroi des ampoules spermathècales est très souvent vacuolisée et cassće. Cette espèce n'est pas toujours facile à distinguer de $S$. heringianus surtout par exemple en ce qui conceme les exemplaires mutilés. Le bulbe pénien est un caractère propre de S. brachystilus, mais selon Cook $(1967: 360)$ S. heringianus présente aussi \& a thick ring of muscle... round the base of the penis which appears to be continuous with the circular layer of the body wall ". Sur la plupart de nos exemplaires ce caractère est aussi très remarquable. Donc, il nous semble important de souligner dans la diagnose de $S$. brachystilus la forme conique, la petite taille et le diamètre du pénis par rapport au bulbe, pour bien distinguer cette espèce de $S$. heringianus. Nos exemplaires présumés de $S$. brachystilus présentent une formation musculeuse à la partie ectale de l'atrium (fig. 4C) qui ne correspond pas avec le bulbe et le pénis décrits par Hrabe (1929), Kasprzak (1973) et Timm (1979) chez S. brachysitus. En conséquence, nous pensons qu'il s'agit d'exemplaires de $S$. heringianus avec l'appareil reproducteur en cours de résorption et ayant perdu la partie distale du pénis, probablement par autotomie.

\section{ANOMALIES}

- Un exemplaire avec une moitié anormale : deux atria dans les segments IX et X, spermathèques en VIII, deux paires de testicules en VIII et IX. deux paires d'ovaires en $\mathrm{X}$ et XI et deux paires d'entonnoirs femelles (Localité : Gerrikaiz, R. Lea, Bizkaia, 30TWN 33 90, 24, nov. 1984).

- Un autre exemplaire avec un atrium qui porte deux pénis, l'autre atrium étant normal. (Localité : Yurre, R. Indusi, Bizkaia, 30TWN 185 786. 15. Nov. 1985). Ce phénomène tératologique, consistant en la présence de pénis surnuméraires, a été déjà décrit par Giani \& Martinez-Ansemil (1983); il s'agissait 


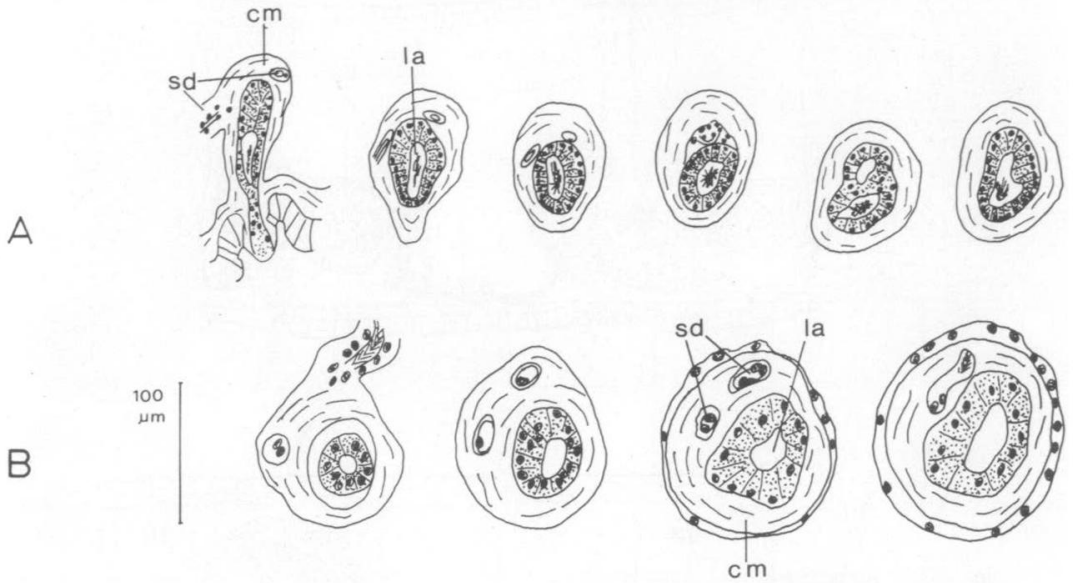

Fig. 3. Stylodrilus lemani (Gr.). A et B : deux séries de coupes histologiques de l'atrium. cm : couche musculaire, sd : sper. miducte, la: lumière atriale.

d'un pénis en $Y$, bifide, et deux pores mâles pour les deux branches. Voir aussi Timm (1979).

Stylodrilus temani (Grube, 1879)

Fig. 3 et $4 A$.

\section{REMARQUES ANATOMIOUES}

Longueur : 15-25 mrn. Diamètre (au niveau du clitcllum) : 0.5-0.8 mm. 15 à $25 \mathrm{~mm}$. Nombre de segments : 64-77. Double annulation à partir du seg. ment V. Soies à pointe simple, plus courtes dans la partie antérieure $(140-170 \mu \mathrm{m})$ que dans la partie postérieure (jusqu'à $190 \mu \mathrm{m}$ ). La taille des soies postérieures se réduit progressivement vers l'arrière. Diamètre des soies environs $5 \mu \mathrm{m}$.

Les glandes pharyngiennes occupent les segments IV à VI, mais dans ce dernier elles sont réduites à la portion ventrale. Les cellules chloragogènes débutent sur le segment VI. La position des gonades et de l'appareil reproducteur coüncide avec celle des descriptions de Hrabe (1970) et Cook (1971). Le clitellum s'étend de la moitié de IX au segment XIII. La vésicule séminale peut atteindre le segment VIII vers l'avant et XV vers l'arrière. L'ovisac peut at teindre le segment XVII.

L'atrium est tubulaire (329-447 $\mu \mathrm{m}$ de long et $85-91$ $\mu \mathrm{m}$ de diamètre), avec une couche musculaire de 14 à $26 \mu \mathrm{m}$ d'épaisseur. Les cellules prostatiques forment une vaste couverture autour de l'atrium. Les spermiductes ont environ $300 \mu \mathrm{m}$ de longueur et $14-20 \mu \mathrm{m}$ de diamètre. Le postęrieur pénètre en XI où il forme une boucle. Ils débouchent dans l'atrium basalement, au tiers de la hauteur de l'atrium. La figure 3 montre le parcours des canaux déférents dans la couche musculaire de l'atrium sur deux séries de coupes histologiques longitudinales. On peut voir l'union des conduits juste avant de déboucher dans la lumière de l'atrium. Il existe un pénis court $(20-47 \mu \mathrm{m})$.

Les spermatheques sont formées par un canal musculeux (294-327 $\mu$ de long) et une ampoule (282-472 $\mu$ de long, 182-236 $\mu \mathrm{m}$ de diamètre).

\section{Discussion}

La description ces dernières années de Stylodrilus longiatriatus Dembitsky, 1976 et S. glandulosus 



Fig. 4. Schémas de l'appareil reproducteur de : A : Stylodrilus lemani (Gr.). B : S. heringianus Clap. typique, C : S. heringianus Clap. à pénis court. a : at rium, sp : spermathèque, $0:$ ovaire, $t:$ testicule, sd : spermiductes, $p:$ pénis, pr: glandes prostatiques, es : entonnoir seminal, ef : entonnoir femelle. 
Giani et Martinez Ansemil, 1984 semble avoir mis fin à la discussion sur la validité du genre $B$ yhonomus et son inclusion dans Stylodrilus (Hrabe 1929. Brinkhurst 1965. Hrabe 1970). L'union des spermiductes avant de s'ouvrir dans la lumiere atriale a été décrite chez Stylodrilus asiaticus (Mich., 1901) (cf. Hrabe, $1970: 289-290$ ). Les descriptions de $S$. minutus Hrabe 1970 et de S. opisthoannulatus (Izossimov 1962) (Hrabe 1970) ne permettent pas de sàvoir s'il se produit le mẽme phénomène d'union.

\section{ANOMALIES}

Un exemplaire dont l'appareil génital sur un côté est déplacé vers l'arrière, avec les spermathèques en $X$ et ke pore mâle en XI. (Localité : Ezkurra, R. Ezkurra, Bidasoa, Navarra, 30 TWN 968 709. 29. avr. 1986).

Stylodrilus parvus (Hrabe et Cernosvitov, 1927) Fig. 5A

\section{REMAROUES ANATOMIOUES}

46-80 segments. Longueur du corps : environ 15 mm et diamètre 0.2 à $0.6 \mathrm{~mm}$. La double annulation débute en III mais elle devient très nette à partir de IV seulement. Soies bifides tout au long du corps, 88-103 $\mu \mathrm{m}$ de long.

Les glandes pharyngiennes sont situées sur les segments IV à VI en position dorsale et ventrale par rapport au tube digestif et uniquement en position ventrale sur le segment VII. Les cellules chloragogènes ne sont pas très allongées et débutent en $\mathrm{V}$.

La position des gonades et de l'appareil reproducteur coïncide avec la description originale de l'espèce. Le clitellum s'étend de IX à XII et généralement $i$ est très peu apparent. A trium subsphérique ou globulaire (73-94 $\mu \mathrm{m}$ de long et 52-61 $\mu \mathrm{m}$ de diamètre). La couche musculaire est fine (5-5.8 $\mu$ d'épaisseur). Les spermiductes pénétrent apicalement dans l'ampoule atriale. Le spermiducte postérieur pénètre dans le segment XI où il forme une boucle. Les cellules prostatiques couvrent la partie apicale de l'atrium. Il n'existe pas de pénis. Les spermathèques ont des ampoules volumineuses (200-250 „ $\mathrm{m}$ ), généralement repliées vers l'arrière, et des canaux relativement courts (73-130 $\mu$ m de long).

\section{ANOMALIES}

Lin individu présentait une double annulation à partir du segment III, des glandes pharyngiennes en V-VII, les pores de la spermathèque situés en VIII avec l'ampoule remplie de sperme en IX, l'atrium et le pore mâle en IX. Il s'agit probablement d'un exemplaire qui a régéneré incomplètement la partie antérieure du corps.

Trichodrilus strandi campoyi n. ssp. Fig. 5B et 5C.

Localites : Gerrikaiz, R. Lea, Birkaia, 30TWN 3390 (6. Dec. 82) (6. mai. 86) / Brinkola, R. Urola, Gipuzkoa, 30TWN 54 62 (20. nov. 81)/Landeta, R. Ibai-Eder, Gipuzkoa, 30TWN 6080 (29 nov, 81)/Usategieta, R. U rumea, Gipuzkoa, 30TWN 9271 (26. nov. 81)/ Arrazua, R. Golako, Bizkaia, 30TWN 287957 (29. nov. 85) / Markina, R. Etxebarria, Bizkaia, 3OTWN 433896 (27. nov, 85) / Ventas, R. Bidasoa, Navarra, 30TXN 224815 (30. avr. 85) / Ezkurra, R. Ezkurra, Navarra, 30TWN 968709 (13. nov. 85) (18. jui. 85) (29 avr. 86) / Sumbilla, R. Bidasoa, Navarra, 30 TXN 086840 (30. avr. 86) / Astigarraga, R. Agaunza, Gipuzkoa, 30 TWN 670579 (28. Avr. 86) / Betelu, R. A raxes, Navarra, 30TWN 843638 (16 jui. 85) (13 nov. 85) (29. avr. 86).

\section{DiAGNOSE}

Longueur du corps : environ 8-10 mm. Diamètre : $0.4 \mathrm{~mm}$. Nombre de segments : 40-50. Il existe une double annulation à partir du segment $V$, d'abord très faible, puis très nettè à partir de VI. Soies bifides, avec la dent supérieure très réduite. La longueur des soies mesurée sur 3 exemplaires est de $89.4 \mu \mathrm{m}$ dans les faisceaux dorsaux antérieurs, de 74-109.4 ${ }^{\mathrm{m}} \mathrm{m}$ dans les faisceaux ventraux antérieurs el de $80-90.5 \mu \mathrm{m}$ dans les faisceaux postérieurs. Le clitellum s'étend depuis la moitié du segment IX jusqu'à la fin du segment XI.

Les glandes pharyngiennes sont disposées dorsalement et ventralement sur les segments IV à VI. Les cellules chloragogènes débutent en VI. La position des gonades et de l'appareil reproducteur coïncide avec la description originale de Hrabe (1936).

La vésicule séminale s'étend vers l'avant jusqu'au segment VI et vers l'arrière jusqu'à XIV. L'atrium est globulaire $(120-173 \mu \mathrm{m}$ de long et $67-100, \mu \mathrm{m}$ de diamètre) et il n'existe pas de canal éjaculateur. La couche musculaire de la cavité atriale est très fine ( $2.9 \mu \mathrm{m}$ d'épaisseur). Les spermiductes ont environ $250 \mu \mathrm{m}$ de longueur et un diamètre de $26 \mu \mathrm{m}$. Ils entrent en contact avec l'atrium vers la moitié de sa 

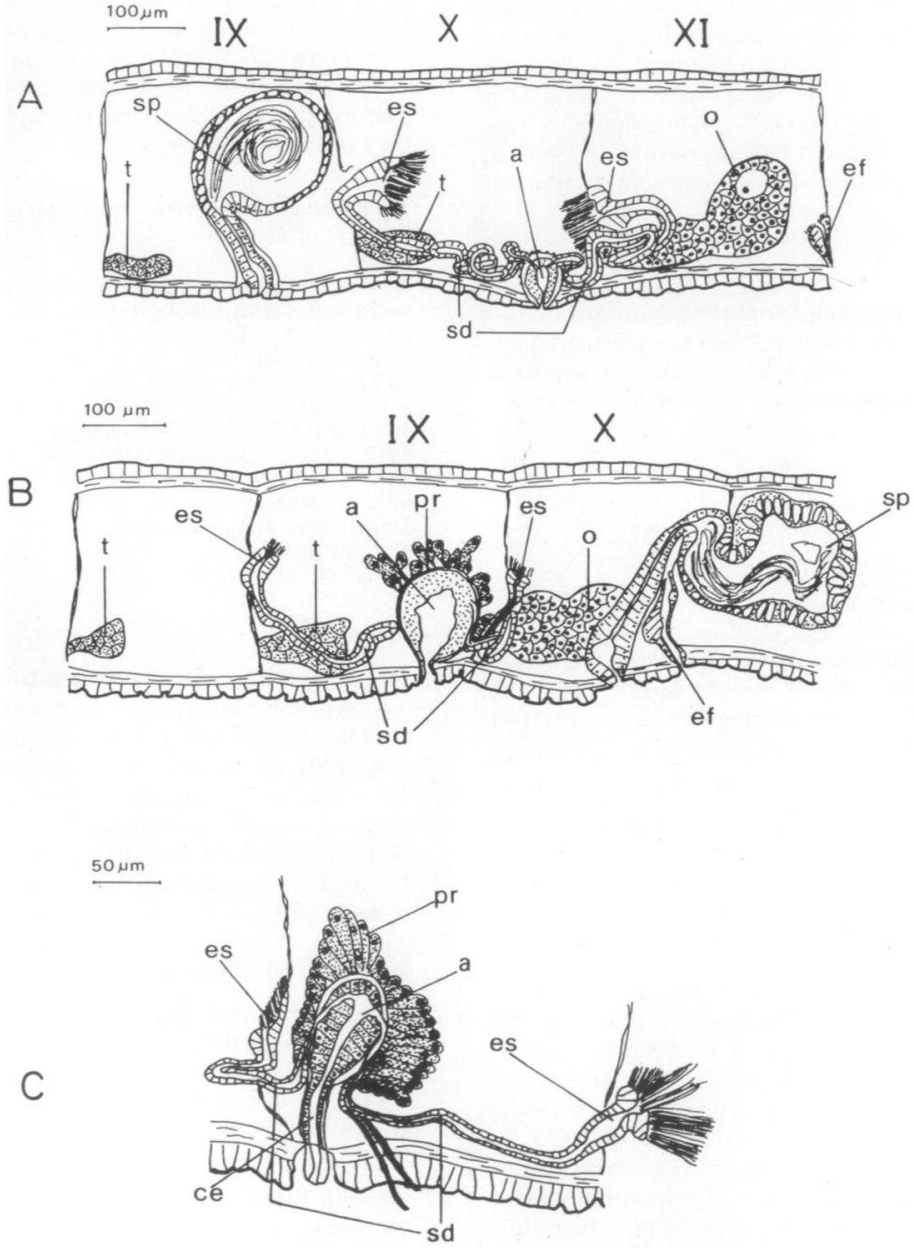

Fig. 5. Schémas de l'appareil reproducteur de : A : Stvlodrilus parvus $\mathrm{Hr}$, et Cer., B : Trichodrilus strandi campoyi n. ssp.r C: Trichodrilus st randi strandi Hr. (dessiné à partir du matériel français de la collection de N. Giani). a : atrium, o : ovaire, $t$ : testicule, ef : entonnoir femelle, es : entonnoir seminal, sd : spermidcute, sp : spermathęue, ce : canal ejaculateur. 
longueur. A partir de là, ils montent à l'intérieur de la couche musculaire de l'atrium, vers la partie apicale, où ils débouchent dans la lumière de l'ampoule Le spermiducte postérieur pénètre dans le segment $\mathrm{X}$. Les cellules prostatiques couvrent la paroi de l'a trium, ou bien complètement, ou bien uniquement sur la partie apicale.

Les pores spermathécaux se trouvent sur la ligne des soies ventrales, en arrière des faisceaux de soies du segment $X$. Le canal de la spermathèque (132-255 $\mu \mathrm{m}$ de long) est renflé dans sa partie ectale, à côté du pore. Les ampoules (149-223 $\mu \mathrm{m}$ de long et 102- $\{70 \mu \mathrm{m}$ de diamètre) occupent les segments $\mathrm{X}$ et/ou XI. Elles peuvent loger ensemble dans le même segment ou bien chacune dans un segment différent. La paroi de l'ampoule peut être très vacuolisée avec des spermatozoïdes à l'intérieur des vacuoles. Le sperme est accompagné par du matériel indéterminé qui ne se colore pas avec l'hématoxyline.

\section{DISCUSSION}

A notre connaissance $T$. strandị n'a été signalée qu'en Yougoslavie (Hrabe 1936) et en France (Giani 1979). Les populations étudiées dans le Pays Basque présentent une série de caractéristiques qui les distinguent de la forme typique et que nous signalons ci-dessous :

1) Existence d'une double annulation sur nos spécimens (elle est absente sur le matériel yougoslave) ;

2) Absence de formations alaires dans les segments du clitellum;

3) Absence d'un canal éjaculateur sur l'atrium (tandis qu'il est très bien développé sur le matériel yougoslave et français : voir fig. 5C).

4) Les ampoules atriales sont très volumineuses : le double des dimensions du matériel type $(60 \mu \mathrm{m}$ de long et $56 \mu \mathrm{m}$ de diamètre, selon Hrabe 1936).

Trichodrilus st randi occupe une position taxonomique très isolée dans le genre due à la position atypique de l'appareil reproducteur, et le caractère bifide des soies. Seule Trichodrilus diversisetosus Rodriguez, 1986 partage ces caractères. Les différences que nous avons observées entre nos spécimens et la forme type ne nous semblent pas suffisantes pour la création d'une nouvelle espèce et nous proposons la dénomination sous-spécifique de campoyi pour les populations de Trichodrilus strandi trouvées dans les rivières du Pays Basque.
Etymologie: Cette sous-espèce est dédiée à la mémoire de Antonio Campoy, zoologiste et spécialiste en Annélides Polychètes.

\section{Remerciements}

J'exprime ma gratitude à Narcisse Giani (Toulouse), pour les fructueuses discussions à propos des espèces StylodriLus brachystilus et Trichodrilus strandi et pour le leg du matériel de sa collection. Je remercie Miguel Escobedo pour l'aide qu'il m'a apportée dans la traduction du manuscript et Ma. Jesús Bidaurrázaga pour la réalisation des coupes histologiques.

Travaux cités

Brinkhurst (R.O.) 1965. - A revision of the genera Stylodrilus and Bythonomus (Oligochaeta, Lumbriculidae). Proc. Zool. Soc. London, 44 : 431-444.

Cook (D.G.). 1967. - Studies on the Lumbriculidae (Oligochaeta) in Britain. J. Zool. Londom, 153 : 353-368.

Cook (D.G.). 1971. - Lumbriculidae. In : Brinkhurst (R.O.) and Jatmieson (B.G.M) (Eds.) Aquatic Oligochaeta of the World. Oliver and Boyd, Edinburgh. p. 200-285.

Giani (N.). 1979. - Les oligochètes aquatiques du SudOuest de la France. ( $2^{e}$ note). Bull. Soc. Hist. Nat. Toulouse, 115 (3-4): 347-358.

Giani (N.) \& Mart inez-Ansemil (E.). 1983. - Diversos casos teratológicos en Oligoquetos dulceacuicolas. Comunicación a la VI Retnión Bierzal de la R. Soc. Esp. Hist. Nat. (Santiago de Compostela, 13.17 Septiembre, 1983).

Eolmquist (C). 1976. - Lumbriculids (Oligochaeta) of northern Alaska and Northwestern Canada. Zool, Jb. Syst., $103: 377-431$.

Hrabe (S.) 1929. - Zwei neue Lumbriculidaem-Arten, sowie einige Bemerkungen aur Systematik einiger bereits Bekannter. Zool. Anz, 84 : 9.21.

Hrabe (S.). 1936. - Trichodrilus strandi n. sp. ein neuer Vertreter der Höllen Lumbriculiden. Festschr. Prof. Dr. Embrik Strand, Riga, 1 : 404-407.

Hrabe (S.). 1970. - Notes on the genera Stylodrilus and Bythonomus (Lumbriculidae, Oligochaeta). Spisy prir. Fak. Uniu. Brna, 515: 283-309.

Kasprzak (K.). 1973. - Notaki o Faunie Skaposzczetow (Oligochaeta) Polski, I. Fragm. Faun. Warsow, 23 (No. 21) : $405-36$.

Mrazek (A.). 1906 . - Die Geschlechtsverhältnisse und die Geschleschtsorgane von Lumbriculus variegatus Gr. Zool. Jb. (Artat.) Jena, $23: 381-462$.

Sokolskaya (N.L.). 1976. - Data on the Lumbriculidae (Oligochaeta) fauna of the Chukchi Peninsula. Bull. Moscow Nat. Soc. (Biol. Sect.), 83 (3) : 43-53.

Timm (T.). 1979. - The Estonian Lumbriculidae. East $i$ NSV Teadoste Akad. Toimetised (Biol), 28 (3) : 158-171. 